\title{
ECOLOGICAL REHABILITATION AND PUBLIC PARTICIPATION: GENERAL CONSIDERATIONS AND EMPIRICAL EVIDENCE FROM A CREEK REHABILITATION SCHEME NEAR COLOGNE, GERMANY
}

\author{
Boris Braun and A. Z. M. Shoeb ${ }^{1^{*}}$ \\ Institute of Geography, University of Cologne, Germany \\ ${ }^{1}$ Present address: Department of Geography and Environmental Studies, University of Rajshahi, Rajshahi \\ 6205, Bangladesh \\ *Corresponding author (email: azms52@gmail.com)
}

\begin{abstract}
Ecological rehabilitation of rivers and streams has become a common practice in environmental planning and water management throughout Europe. With regard to rehabilitation projects public participation and bottom-up planning processes are favoured by state and local regulations alike. However, there are mixed experiences about public support of rehabilitation schemes. Whereas people support environmental improvements on a general level, acceptance is dwindling when conflicts of use arise or access to certain areas is going to be restricted. This study focuses on the assessment of public attitudes to rehabilitation and on improving the understanding of people's preferences. The results of a representative questionnaire survey conducted in three villages along a rehabilitated creek in a densely populated suburban area near Cologne show that local support for rehabilitation is generally high. More than two thirds of all residents support the rehabilitation scheme even when considering the costs involved. This support, however, varies between different age groups, with young adults being more sceptical than older people. The reasons for the fact that the younger generation is less supportive with regard to rehabilitation schemes might be related to their lesser interest in local affairs in general as well as their involvement in leisure activities that cannot be performed in rehabilitation sites along a small creek. Moreover, the findings reveal that many people want to have unlimited access to rehabilitated areas for recreational activities. This reflects a trend towards the perception of nature as an individual consumer good and has to be considered by environmental managers - not only in Europe, but in other parts of the world as well.
\end{abstract}

Key words: Rehabilitation, restoration, awareness, public participation, river, Germany.

muं vsk: পুরো ইউরোপে নদী-নালার ইকোলোজিকাল পুনরুজ্জীবিতকরণের মাধ্যনে পরিবেশ পরিকল্পনা ও পানি ব্যবস্থাপনা একটি সাধারণ চP园 পরিণত হয়েছে। এটা প্রকল্পায়নে জনগłYর অংশ গ্রহণ এবং “নীচ থেকে উপর” পরিকল্পনা-প্রক্রিয়া পৃষ্ঠপোষকতা পাচ্ছে রাষ্টীয় ও স্থানীয় বিধি-বিধানের মাধ্যমে। এতৎসত্ত্বে, পুনরুত্জীবিতকরণ স্কীম সমজনগłYর মধ্যে মিশ্র মনোভাব দেখা যায়। যদিও পরিবেশ উন্নয়নে জনগনের সাধারণ সমথশ্টি বিদ্যমান, তথাপিও পুরোমাত্রায় গ্রহনীয়তায় ভাটা পড়ে যখন জমি ব্যবহারেi ai łb মতানৈক্য দেখা দেয় এবং প্রবেশাধিকারে বিধি-নিভেধ আরোপিত হয়। এই গবেষণা প্রবłন্ধ নদী-নালা পুনরুজ্জীবিতকরণে এবং এলাকাবাসীদের পছন্দের সমঝোতা উন্নয়ন ইস্যুতে উক্ত এলাকার জাg অিদের দৃষ্টিভঙ্গি মূল্যায়ন করv nq । এটা জাg

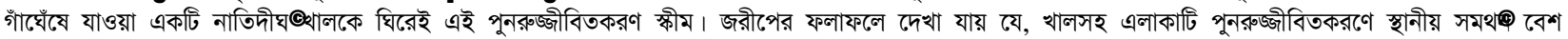
জোরালো। আথিক্টি ব্যয়ভার জড়িত থাকা সত্বেও দুই-তৃতীয়াংশ বাসিন্দা এই প্রকল্পটি সমথత্টি করে। তবে এই সমথচী বয়সভেদে ভিন্নতা আছে। যেমন বয়স্কদের তুলনায় তরুণরা

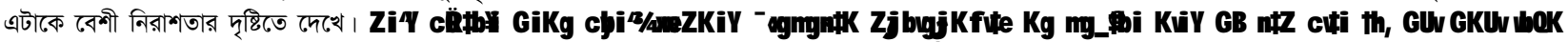

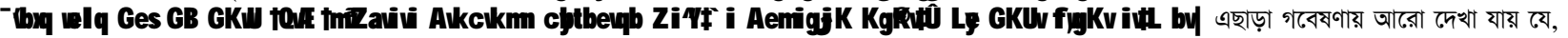
বেশ কিছু সং "ক জনসাধারণ প্রকল্প এলাকায় বিনোদনমূলক কমক্কান্ড করার জন্য সীমাহীন প্রবেশাধীকার আশা করে। এটা এরকম একটা ধারণার প্রতিফলন ঘটায়, যেন প্রকৃতিও আলাদারকম একটা তোগ্যপণ্য এবং পরিবেশ ব্যবস্থাপকদের এটা বিবেচনায় আনতে হবে-শুধু ইউরোপেই নয়, পৃথিবীর অন্যান্য অংশেও ।

\section{Introduction}

Rivers and streams worldwide have been channeled and modified by human activities. In Europe this process started centuries ago when people built water mills and began to use water energy in a systematic way. Especially in the 1950s and 1960s even small rivers and creeks were fundamentally altered in their appearance due to local flood protection schemes and the attempt to gain more land for agriculture, houses, factories, and roads. Many small rivers and creeks were straightened, forced into a concrete bed or even completely tubed. The last few decades, however, have witnessed a marked paradigmatic shift in the practice of environmental planning and river engineering. It has become common practice in Europe and many other parts of the world to allow rivers and creeks more space again and thus to increase the quality of natural habitats. It has become clear that channeled or tubed creeks have lost most of their natural ecosystem functions and provide habitat only for ubiquitous and very adaptable species of plants and animals. Serious problems, for example, are barriers to migration that interfere with, or prevent the upstream or downstream movement of fish or even invertebrates. These obstructions include dams, culverts, and heavily engineered channels of concrete. 
Rehabilitation and revitalization schemes try to bring back basic ecosystem functions to rivers and creeks. A major aim of these efforts is to restore the dynamic of discharge and river bed morphology to allow migration and through-passing of aquatic organisms. Nevertheless it is essential to assure for enough capacity of discharge to meet the requirements of flood protection. River rehabilitation schemes are expected to combine improved flood protection measures with ecological restoration (Boon et al. 2000; European Union 2000).

Restoration projects tend to be, in general, relatively well accepted after completion (Junker and Buchecker 2008; Buijs 2009; Schaich 2009). In counties like Germany, public support is often based on a widespread sense of environmentalism. However, this environmentalism is not unconditional. Resistance against ongoing projects may arise because restorations imply a loss of agricultural land, alter the space for recreational and leisure activities or might be in conflict with flood protection requirements. Environmental protection and ecological restoration might be harder to accept when larger amounts of public spending are involved or respective projects take place close to where people live and work. This raises questions about public support for rehabilitation projects in densely populated and intensively economically utilized areas. To provide some insights into these issues we shall first briefly discuss the history of environmentalism and rehabilitation efforts in Europe and then present findings from an empirical study on a rehabilitation project in the suburban area of Cologne, Germany's fourth largest city with a population of about 1 million.

\section{Background}

\section{Environmentalism in Germany}

In a representative survey conducted on behalf of the Federal Ministry for the Environment it was observed that people realize the loss of biological diversity. A great majority of the respondents considered the loss of biological diversity as a big problem and 95 per cent stated that ecologically intact nature is essential for a good life. 89 per cent considered protection of nature being an important political task in Germany and 68 per cent expressed worries about the quality of nature we inherit to the next generation (BMU and BfN 2010). Moreover, 'nature' has not only an abstract meaning for the German population as 81 per cent of the respondents stated that they are going out to enjoy the beauties of nature at least once a week. These figures clearly indicate that nature and biodiversity nowadays have a high priority for the German population.

People's strong awareness of nature and environmental protection started in the early 1970s when environmentalists began their protests against nuclear power. Later the movement gradually broadened its scope and incorporated ideas on eco-ethics, environment-friendly ecosystems, ecological modernisation, and biodiversity conservation, which finally led to a powerful and politically influential green movement. A very obvious political result of these changes in society was the establishment of the German Greens (Die Grünen) as a political party in 1980. Quickly the new party made its way into local and state parliaments and in 1998 even became a partner of a coalition government on the federal level, making Germany the first country in the world ruled by a social democratic-green alliance. Thus, it would be too shortsighted to reduce the idea of political environmentalism in Germany to the Green party and the green movement alone. Environmental ideas also prevail in other leading political parties, including the Social Democratic Party of Germany (SPD), the Christian Democratic Union (CDU) and the Free Democratic Party (FDP). An important step for environmental politics in Germany was the establishment of the Federal Environment Ministry in 1986 under a conservative coalition government between CDU and FDP. All these symptoms and attributes indicate the rapidly growing public awareness about nature, biodiversity, environmental protection and ecological rehabilitation in Germany (Holdgate 1996; Goodbody 2002).

\section{EU Natura 2000 and Water Framework Directive: from top-down to bottom-up}

Environmental protection is not only a task of national and local governments alone. Today, the European Union (EU) is a major player in the field of nature conservation. The EU's Natura 2000 directive on flora, fauna and habitat is a basic framework for nature conservation in Europe. It is a holistic approach and integrates all stakeholders in order to protect biodiversity. The conceptual core of Natura 2000 is a network of protected areas within Europe ranging from the Canaries to Crete and from Sicily to Finnish Lapland. Through Natura 2000 about 9.3 per cent of Germany's land area are now ecologically protected as so-called FFH (flora, fauna, habitat) areas (BfN 2010). This percentage is below the European average of 13.6 per cent, but slightly higher than that of some comparable industrialized countries (United Kingdom: 7 per cent). 
The Dutch Nature Policy Plan (LNV 1990) has provided a backdrop for formulating basic elements of the Natura 2000. But interestingly, the Netherland's government was at first strongly opposed by local inhabitants of the province of Friesland when in 1995 it planned to turn 550 hectares of pasture into protected wetlands. This strong protest and resistance, especially from the farmer community, became an icon for a widespread protest movement in many parts of the Netherlands as well as in other EU member states. Similarly, Natura 2000 faced almost the same problems during its implementation in many EU member states. Even some of the 'old' EU members such as Denmark, Finland, France, Germany, Greece and Ireland had been taken before the European Court of Justice (ECJ) by the European Commission for failure in implementing some policies in accordance with the Habitat Directive (Paavola 2004). After careful investigation it was observed that the reasons of initial failure of implementing the European nature conservation policy were its strict top-down approach and the predominance of traditional command-andcontrol regulations. Therefore, the EU and almost all member states shifted from a top-down to a bottom-up approach as well as to more participatory and interactive modes of decision making (nature for people, not people for nature) (Van den Belt 2008; Wurzel 2008).

Equally relevant for the rehabilitation of rivers and streams in Germany is the European Water Framework Directive that came into force in December 2000 (European Union 2000). It commits EU member states to achieve good qualitative and quantitative status of all water bodies (including groundwater and coastal waters) by 2015. The directive follows a holistic, participatory and catchment-oriented approach to development with a specific focus on ecological improvements. The directive in particular demands a good ecological status of water bodies which is defined in terms of quality of the biological community, hydrological characteristics and chemical composition. The European member states had to translate the directive into national legislation. This happened in Germany in June 2002 with an amendment of the national water act (Wasserhaushaltsgesetz). Even though protection and rehabilitation of water bodies started much earlier - the first national water act is dating back to 1960 and a paradigmatic shift concerning the discharge of municipal waste water treatment from exclusive protection of human health to the protection of natural ecosystems was happening as early as 1979 (Seeger 1999: 54) - the European Water Framework Directive undoubtedly has had a major impact on water management in Germany. Most prominently, it led to an increasing number of small and large-scale rehabilitation schemes all over the country.

\section{Rehabilitation or restoration?}

The terminology in the field of nature conservation and protection is complex and sometimes confusing. There are more than dozens of terms such as restoration, reclamation, rehabilitation, preservation, conservation, protection, naturalization, each having a slightly different meaning and implication. Two terms, however, are dominating the scene when it comes to the improvement of the ecological quality of rivers and streams: rehabilitation and restoration. Choi (2007) defines 'rehabilitation' as an act of improvement from a degraded state. According to Diggelen et. al. (2001: 116) rehabilitation makes the landscape more 'natural', but it does not necessary include a significant increase in biodiversity. This means that rehabilitation involves the recovery of basic ecosystem functions and processes in formerly degraded habitats, ensuring that the system will remain in a stable ecological state, but it does not necessarily re-establish the pre-disturbance conditions. Rehabilitation, therefore, aims to re-establish the productivity of some, but not necessarily all plant and animal species thought to be originally present at a specific site (Bradshaw 2002).

'Restoration' is more ambitious than rehabilitation, as it implies that the conditions of the site at the time of disturbance will be replicated after a development activity. However, ecological restoration on a grand scale is expensive, time-consuming and, often, simply impossible (Wali 1992). The Society for Ecological Restoration (SER) defines restoration as the process of assisting the recovery of damaged, degraded, or destroyed ecosystems (SER 2002; Hobbs 2004). This definition seems to reiterate the concept of rehabilitation as synthesized by Wali (1992) and Bradshaw (2002). Choi (2007) tries to find a compromise between these two terms and proposes to redefine 'restoration'. In Choi's opinion "the paradigm of ecological restoration needs to be redefined with functional rehabilitation for the future, not nostalgic recompositions of the past. We need to admit our inability to restore an ecosystem to its very original state" (Choi 2007: 351, 352). Mostly, it will be impossible to turn back the wheel and regain the species that inhabited now degraded ecosystems before industrialization or even the beginning of agriculture. Climate, landscape ecology, and man-land interaction have changed dramatically during the course of industrialization and urbanization (Wali 1999; Davis 2000; Cairns 2002; Choi 2004). Native plant and animal species became extinct and invasive alien species have already changed the structure of many European ecosystems (Boehmer 2011; Loos and Schmitt 2011). Moreover, we often do not know the exact species 
assembly that existed before human intervention. So it is apparent that the term 'rehabilitation' is more applicable to most projects, rather than the term 'restoration' in the narrower sense. The case of the Pulheim creek in a densely populated and fundamentally transformed (sub-)urbanized area is no exception from this rule.

\section{Material and Methods}

Pulheim creek rehabilitation scheme

Pulheim creek (locally known as 'Pulheimer Bach') is a short stream in the catchment area of the river Rhine. It is only eight kilometres in length and not more than three meters in width (Fig. 1). According to the European river typology the creek can be classified as a small (catchment $<100 \mathrm{~km}^{2}$ ) lowland river (altitude < $200 \mathrm{~m}$ ). The geology of the catchment area is dominated by Pleistocene terraces which are partly covered by loess deposits. The source of the creek is located on the forestcovered eastern slope of the Glessener Hill (Glessener Höhe) on an altitude of about 100 meters. On its way it passes through mostly open agricultural land, as well as the villages of Glessen (population 2010: 2,421), Sinthern $(3,404)$, and Geyen $(2,421)$ and the district town of Pulheim (21,645 in the core settlement). East of Pulheim the creek percolates to the ground when it reaches gravel deposited by the river Rhine during the later pleistocene period on an altitude of 45 meters (area locally known as Laachen).

Up to the 1980s the Pulheim creek shared the fate of many small creeks in densely populated and agriculturally fertile areas. It was straightened and forced into a concrete bed in order to allow more space for farming activities and the growth of settlements in a period of rapid population growth (figure 2). Ecological balance was disturbed and the creek's concrete bed did not support natural ground-water penetration.

In 2006 the local authority in charge of the creek's management, the 'Unterhaltungsverband Pulheimer Bach' (in short 'Bachverband'), started an initiative to rehabilitate larger sections of the creek and applied for funding from the state government of North RhineWestphalia. The main reason for the rehabilitation scheme was the necessary improvement of water quality, as water from Pulheim creek is used for the drinking water supply of Cologne City. Other reasons include the overall ecological improvement of the area, the provision of opportunities in environmental education, and the creation of attractive green spaces for leisure activities. Moreover, the rehabilitation scheme is expected to improve downstream flood protection by slowing down discharge after heavy rainfall. The plan was to rehabilitate the creek wherever possible and to link the rehabilitated sites by a sign-posted nature trail.

After receiving a sum of Euro 155.000 from the state government and a respective co-financing of Euro 200.000 from the two municipal councils involved (Cities of Pulheim and Bergheim), the rehabilitation work commenced in late 2007/early 2008 in the Bachaue area east of Geyen (Fig. 1). Work on another section east of Glessen started in mid-2010 immediately after the completion of the questionnaire survey. Structural work has been accomplished by a landscape engineering firm and comprised the removal of concrete beds, tubes and other technical migration barriers. These concrete structures have been replaced by a meandering bed of gravel. Moreover, native trees such as black alder (Alnus glutinosa), reed, and hydrophytes such as different species of water lilies, hard rush (Juncus inflexus) and butterbur (Petasites hybridus) have been planted on the banks of the creek (figure 3). After a rather short time amphibians, insects and even fish species re-colonized Pulheim creek, most prominently the lively three-spined stickleback (Gasterosteus aculeatus). Other animal species such as the endangered European crayfish (Astacus astacus) have been successfully reintroduced.

From an ecological and aesthetical viewpoint the rehabilitation scheme is thus certainly a success. But how does the local population participate in the scheme and how do people perceive and assess what has happened?

\section{Methods}

In order to understand more about awareness and perception of the rehabilitation scheme among the local population, a standardized questionnaire survey was conducted in three villages along the creek between September 2009 and June 2010. The three villages are Glessen (population 2010: 2,421), Sinthern $(3,404)$ and Geyen (2,421). The villages are located along the creek at altitudes of 90 metres (Glessen), 75 metres (Sinthern) and 60 metres (Geyen). Glessen (study area 1) is located on the foot of the forest-covered Glessener Hill in a rather scenic setting. Further downstream the landscape around Sinthern (study area 2) and Geyen (study area 3) is less attractive and green space is more limited. However, because of their proximity to Cologne and excellent road connections, Sinthern and Geyen are still popular suburban residential areas. 


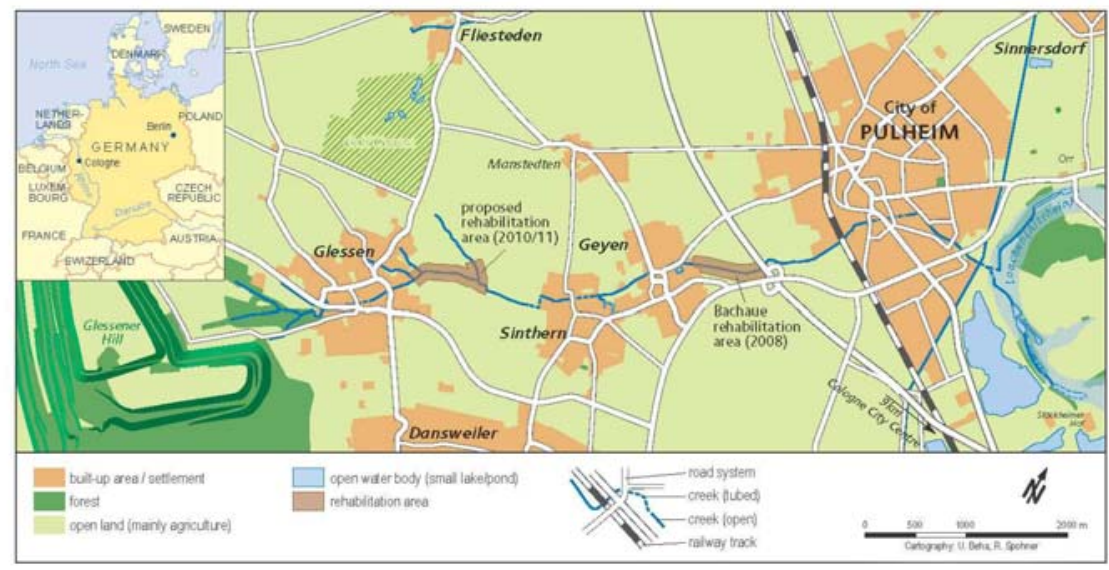

Fig. 1. Pulheim creek area near Cologne, Germany

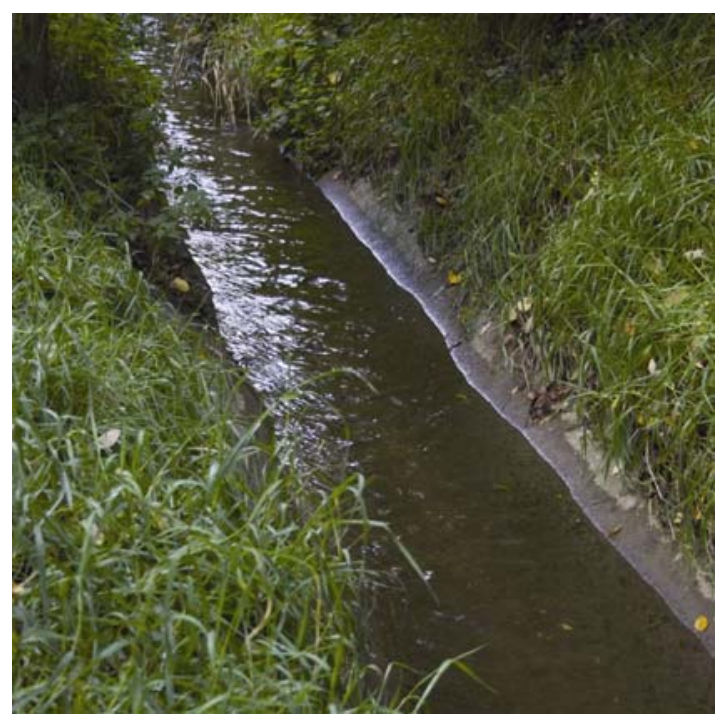

Fig. 2. Pulheim creek east of Geyen (Bachaue) before rehabilitation in summer 2007 (Photo courtesy: Reinhard Zeese)

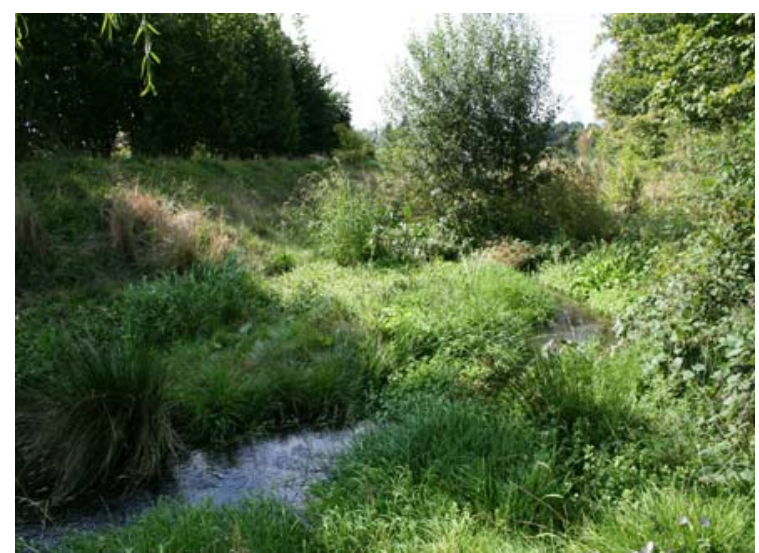

Fig. 3. Pulheim creek east of Geyen (Bachaue) after rehabilitation in summer 2009 (Photo courtesy: Boris Braun) 
The interviews were conducted with the help of students and took about 15 to 30 minutes each. 719 households participated in the survey. All respondents were 16 years or older and interviewed face-to-face at their home via a standardized questionnaire with 29 questions. The survey covers about 8 per cent of the adult population living in the three villages. Non-parametric tests indicate that the sample is not biased to any considerable degree. Distribution of men and women, different age groups and spatial sectors within the villages are almost equal in sample and population. Thus, the sample can be regarded as representative of the resident population of the three villages aged 16 years or older. All data analysis was carried out using the Statistical Package for the Social Sciences (SPSS).

Gender, age and household characteristics of the respondents very much reflect the population and household structures of suburban environments in Germany. Among the interviewees 55 per cent are women and 45 per cent men; the median age is 49 years (range: 16 to 89 years), and the average household size is 2.8 (range: 1 to 7 members per household). Very few households comprise of more than two generations (parents and children), and households with more than two dependent children are relatively rare. They account for only 4 per cent of all households. Most families live in detached or semi-detached one-family dwellings (89 per cent).

\section{Results and Discussion}

Awareness, participation and support: Empirical evidence

There is a high awareness of the rehabilitation projects along Pulheim creek. The rehabilitation scheme is well known to most people in the area. Almost two thirds of the respondents knew about the rehabilitation programme and already visited rehabilitated sections of the creek (Table 1). Fifty three per cent of the respondents also are satisfied with the level of information they received on the programme (Table 2). These shares are even higher in the village of Geyen (study area 3) which is located close to the major and most prominent rehabilitation project along the creek (74 and 70 per cent respectively). Obviously geographical proximity to the place, where the rehabilitation actually happens, matters in this regard, despite an extensive coverage of this topic in local newspapers and information available through the internet. This is also highlighted by the fact that inhabitants of Geyen are visiting the rehabilitated creek significantly more often during their leisure time as compared to the residents of the other two villages. More than half of the respondents in Geyen (study area 3) stated that they visit the 'Bachaue', the rehabilitation area close to their village at least once or twice a month; 21 per cent even come several times a week, mostly going for a walk (often with pet dogs) and/or simply enjoying nature.

Before rehabilitation took place here, interest in this area was considerably lower. Only 44 per cent of the respondents in Geyen (study area 3) stated that they visited the area before rehabilitation, as compared to 75 per cent afterwards. Obviously, the rehabilitation of the 'Bachaue' has brought the area back into people's minds and considerably increased their interest in visiting the area. However, the geographic range of these effects is limited. Inhabitants of Sinthern (study area 2) and Glessen (study area 1) show considerably less interest in the rehabilitated area. From this it can be concluded that the ongoing changes in one's immediate neighbourhood substantially increases one's level of awareness and support. People very much support the changes and environmental improvements that are taking place, but they want to be given an opportunity to experience and enjoy these achievements in close reach.

Table 1. Perception of the rehabilitation scheme by local population

\begin{tabular}{|l|l|c|c|c|}
\hline \multicolumn{2}{|c|}{ Study area } & \multicolumn{2}{c|}{$\begin{array}{c}\text { Do you know about } \\
\text { the rehabilitation } \\
\text { programme? }\end{array}$} & \\
\cline { 3 - 4 } \multicolumn{2}{|c|}{} & Yes & No & Total \\
\hline $\begin{array}{l}\text { Village of } \\
\begin{array}{l}\text { Glessen } \\
\text { (Study area 1) }\end{array}\end{array}$ & $\mathrm{N}$ & 187 & 123 & 310 \\
\hline $\begin{array}{l}\text { Village of } \\
\text { Sinthern } \\
\text { (Study area 2) }\end{array}$ & $\mathrm{N}$ & 60.3 & 39.7 & 100.0 \\
\cline { 2 - 4 } $\begin{array}{l}\text { Village of Geyen } \\
\text { (Study area 3) }\end{array}$ & $\mathrm{N}$ & 131 & 83 & 214 \\
\hline \multicolumn{1}{|c|}{ Total } & $\mathrm{N}$ & 61.2 & 38.8 & 100.0 \\
\cline { 2 - 4 } & $\%$ & 74.2 & 25.8 & 100.0 \\
\hline
\end{tabular}

Source: questionnaire survey 2009/2010; Chi square: $p=0.003$; $\mathrm{N}=$ number of respondents.

Table 2. Satisfaction with information received on rehabilitation projects

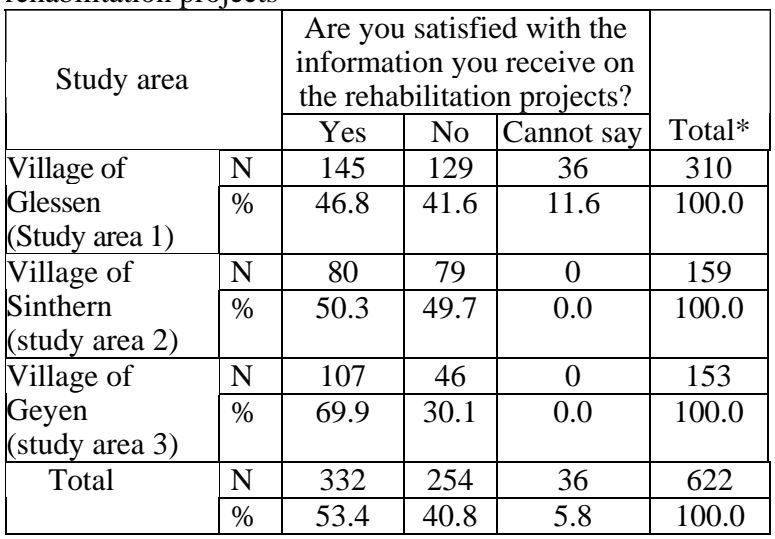

Source: questionnaire survey 2009/2010; Chi square: $\mathrm{p}=0.000$; * 97 interviewees did not respond to this question; $\mathrm{N}=$ number of respondents. 
Local support for the continuation of the rehabilitation programme to continue is strong. 89 per cent of all interviewees stated that they generally support the idea to extend the rehabilitation programme to other stretches of the creek. Yet, rehabilitation is not a 'free lunch'. It costs money that taxpayers have to pay and that is no longer available for other public investments (opportunity costs). Therefore, the interviewees have been in a more specific way if they think that environmental rehabilitation is a reasonable public investment even though rehabilitation of a stretch of about 500 meters costs about Euro 300,000. Not surprisingly, the support dropped somewhat when confronted with the costs of environmental improvement, but still 68 per cent of the respondents were in favour of a continuation of the rehabilitation scheme, stating that this is worthwhile public spending (table 3). In all the three study areas a clear majority of people is positive, but in Geyen (study area 3) - the village closest to the already rehabilitated sections of the creek - support for public spending is significantly higher (83 per cent) as compared to Sinthern and Glessen (67 and 60 per cent, respectively).

Table 3. Support for public spending for rehabilitation of Pulheim creek (as \%)

\begin{tabular}{|c|c|l|c|c|c|c|}
\hline \multirow{2}{*}{$\begin{array}{c}\text { Study } \\
\text { areas }\end{array}$} & \multicolumn{4}{|c|}{$\begin{array}{c}\text { Do you think spending taxpayer's money } \\
\text { for rehabilitation is worthwhile? }\end{array}$} & \multirow{2}{*}{ Total } \\
\cline { 2 - 6 } & $\begin{array}{c}\text { Strong } \\
\text { support }\end{array}$ & Support & $\begin{array}{c}\text { Undec } \\
\text { ided }\end{array}$ & $\begin{array}{c}\text { Scepti } \\
\text { cal }\end{array}$ & $\begin{array}{c}\text { Very } \\
\text { sceptical }\end{array}$ & \\
\hline Glessen (1) & $30 \cdot 6$ & 29.6 & $24 \cdot 3$ & 7.7 & 7.7 & 100.0 \\
\hline Sinthern (2) & $31 \cdot 8$ & 35.2 & 22.9 & 3.9 & 6.1 & 100.0 \\
\hline Geyen (3) & $43 \cdot 8$ & 39.4 & 9.4 & 3.1 & 4.4 & 100.0 \\
\hline Total & $34 \cdot 3$ & 33.7 & 20.1 & 5.5 & 6.4 & 100.0 \\
\hline
\end{tabular}

Source: Questionnaire survey 2009/2010

In order to get a deeper insight into the opinions of the local population it was also asked how people assess the rehabilitation according to four major objectives of the scheme. These objectives were (1) 'environmental conservation', (2) 'increasing recreational value', (3) 'beautification of landscape', and (4) 'flood prevention and mitigation'. The results are presented in table 4. It is obvious from the data that the local population indeed has a very high opinion of the rehabilitation of Pulheim creek, especially when it comes to core objectives like environmental conservation or the quality of landscape values. The recreational value of the rehabilitated sites is also assessed rather positively, but to a somewhat lower degree. The reason for this might be that the rehabilitated sites are small in their spatial extent (between 100 and 500 meters along the creek) and therefore, inevitably, provide only limited possibilities for recreational activities. Some popular leisure activities like inline skating, fishing, camping or motorized sports are not possible. Others - for example biking or horse riding - are limited to some sections along the creek where an appropriate infrastructure exists.

Table 4. Assessment of rehabilitation along Pulheim creek with regard to major objectives (as \%)

\begin{tabular}{|c|c|c|c|c|c|c|}
\hline Objectives & $\begin{array}{c}\text { Very } \\
\text { positive }\end{array}$ & Positive & $\begin{array}{c}\text { Undec } \\
\text { ided }\end{array}$ & $\begin{array}{c}\text { Negat } \\
\text { ive }\end{array}$ & $\begin{array}{c}\text { Very } \\
\text { nega } \\
\text { tive }\end{array}$ & Total \\
\hline $\begin{array}{c}\text { Environmental } \\
\text { conservation }\end{array}$ & 63.6 & 31.2 & 3.9 & 1.1 & 0.2 & 100.0 \\
\hline $\begin{array}{c}\text { Recreational } \\
\text { value }\end{array}$ & 52.9 & 37.5 & 7.5 & 1.8 & 0.3 & 100.0 \\
\hline $\begin{array}{c}\text { Landscape } \\
\text { beautification }\end{array}$ & 64.4 & 31.0 & 4.1 & 0.3 & 0.2 & 100.0 \\
\hline $\begin{array}{c}\text { Flood } \\
\text { prevention }\end{array}$ & 38.7 & 45.0 & 11.2 & 4.5 & 0.6 & 100.0 \\
\hline
\end{tabular}

Source: Questionnaire survey 2009/2010

A more controversial point is the nexus between rehabilitation and flood control as the latter was one of the main reasons for the authorities to put the creek into a concrete bed back in the 1960s. There is no scientific proof that rehabilitation is likely to increase flood risks after torrential rain. On the contrary, if anything, rehabilitation increases infiltration rates and prevents downstream areas from becoming flooded. Nevertheless, some of the older inhabitants of the area are still a bit sceptical about the impact of rehabilitation on floods. Moreover, flood control is a complex issue which is difficult to understand in its full implications. Accordingly, a substantial number of interviewees (344 respondents) indicated that they feel not able to answer this question. Yet, it can be concluded that people's opinions are predominantly positive with regard to the effects of rehabilitation on flood prevention.

In recent times, the rehabilitated stretches of Pulheim creek have also been incorporated in the curricula of local schools - primary and secondary. Schools have established so-called 'green classrooms' (Grünes Klassenzimmer) at various locations. These are environmentally friendly outdoor learning arrangements where teachers go with their biology or geography classes on a regular basis to study the ecology of the creek as well as its flora and fauna. Primary school kids measure water temperatures or observe plants in order to get an understanding of seasonal variations of the creek's ecology. Students of secondary schools, for example, are more concerned with the analysis of water 
pollutants or the classification of animals and plants. The 'green classroom' is seen as a vehicle for promoting conservation projects to children and to enhance their understanding of nature (environmental education). Teachers as well as the students and their parents are very positive about the 'green classrooms' that have been established. From an educational viewpoint the 'green classroom-initiative' is undoubtedly a great success, but it is also a good way to convey the message of environmental protection and rehabilitation to the general public. As the survey reveals, about 30 per cent of all interviewees have already heard about the 'green classroom initiative' - not only parents of school children, but also their grandparents and people that read something about it in local newspapers. People's opinion on the 'green classroom initiative' is overwhelmingly positive: 98 per cent of all respondents that knew about the 'green classrooms' also supported the idea behind it (81 per cent 'very strongly' and 17 per cent 'strongly').

\section{Bivariate analysis: Variations of participation and support}

As the data show, the rehabilitation scheme on Pulheim creek receives a lot of support from the local population. However, it may be that different segments of the population have slightly different opinions on the issue. To control for this we applied chi-square testing as well as comparison of means and t-testing. Through these tests we have been able to analyze the impacts of individual characteristics such as gender, age, and household structures on the degree of support that the rehabilitation scheme receives. The major result of these analyses is that individual characteristics slightly vary some findings, but do not have a decisive impact on the opinions and attitudes of the overall people. This is consistent with the results of Schaich's research on restoration of river floodplains in Luxembourg (Schaich 2009). His study showed that basic beliefs, lifestyle concepts and environmental behaviour are more important determinants of individuals' perceptions of floodplain restoration measures than simple sociodemographic.

Almost completely irrelevant is the fact if respondents have dependent children or not. This factor correlates with none of the variables describing the opinion on or the knowledge of rehabilitation issues, except the level of knowledge people have on the 'green classroom initiative' which is higher among the parents (40 per cent as compared to non-parents 23 per cent) ( $\mathrm{p}=0.002)$. Gender has a slightly higher impact on attitudes towards rehabilitation issues than parenthood. In general, women are more positive and supportive than men, especially with respect to the aspects of environmental conservation and flood prevention. These differences are, however, not statistically significant $(\mathrm{p}=0.116$ and $\mathrm{p}=0.127$ ). The same is true for the slightly higher awareness towards rehabilitation projects in general $(p=0.144)$ and the knowledge about green classrooms $(p=0.143)$. Rather surprisingly, women are somewhat more sceptical than men when it comes to spending public money for rehabilitation projects. This relation is statistically significant $(\mathrm{p}=0.028)$.

The only factor (among the factors we analyzed) which is really influential when it comes to attitudes towards rehabilitation is 'age'. People in all age brackets generally support the idea of rehabilitation, but nevertheless the age of interviewees significantly correlates with a whole range of other aspects. Elderly people $\left(65^{+}\right)$, for example, are more sceptical about the impacts rehabilitation might have on floods, some of them still remembering incidents of severe flooding before structural mitigation measures such as retention ponds have been built $(\mathrm{p}=0,048)$. Apart from this, elderly people, but also people in the age bracket 40 to 65 , are the strongest supporters of the rehabilitation scheme and also the best informed. Younger people (1620 years of age) are slightly more sceptical and significantly less well informed about specific rehabilitation projects along Pulheim creek. For example, only 36 per cent of the respondents under the age of 21 knew about rehabilitation scheme before the interview took place, as compared to more than 70 per cent of the people older than $40(\mathrm{p}=0,000)$. Similarly, the younger generation is rating the recreational and the aesthetical value of rehabilitated sites less highly $(p=0,001$ and $p=0,000)$ and is also more sceptical towards rehabilitation in general $(\mathrm{p}=0,008)$ and public spending in particular $(\mathrm{p}=0,001)$. The possible reasons for the fact that the younger generation is less interested, less informed and slightly less supportive with regard to the rehabilitation scheme are manifold. A generally lesser willingness to read local newspapers and a lesser interest in local politics certainly plays a role in this regard, as does the fact that many leisure activities popular with young people cannot be performed in rehabilitation sites along a small creek. This does not mean, however, that the younger generation is less interested in ecological problems and environmental conservation overall. The possible impacts of climate change and environmentally sensitive behaviour are very important issues for many young Germans as the most recent 'Shell Study' indicates (Shell Deutschland 2010). What is less prevalent in this generation, though, is the willingness 
to participate in local affairs as well as in local politics and local action groups.

For the authorities responsible for the rehabilitation of Pulheim creek the message from these findings is clear. They have to address young people in a more targeted and more effective way than they did in the past. A better and more adequate communication is certainly the best chance to raise interest and to establish higher rates of participation among young adults.

\section{Nature as 'consumer good' - empirical evidence}

The somewhat limited interest of the younger generation in local rehabilitation projects can be regarded as a reflection of broader changes in western societies. On the one hand, people are aware of ecological problems and willing to give political priorities to environmental concerns. On the other hand, large parts of the society are dominantly consumption-oriented and increasingly hedonistic. People have more leisure time due to a reduction of working hours and increasing income levels have modified traditional consumption patterns. In a postmodern consumer society nature itself, or more precisely an intact natural environment, is increasingly regarded as 'consumer good'. Nature is becoming a stage for self-fulfilment, a playground for sporting activities and entertainment (BfN 2006). People support the idea of environmental protection, but at the same time they want to be given the opportunity to access and to experience intact nature and its beauties.

This leads us back to an aspect which has already been raised earlier but did not elaborate further. The empirical findings very much support the notion of nature as 'consumer good'. When people were asked to give suggestions for improvements of rehabilitated areas along Pulheim creek many respondents demanded more 'facilities' such as better footpaths, more picnic areas, park benches and even garden restaurants. With regard to the proposed rehabilitation site east of their village the respondents in Glessen (study area 1) were given the possibility to choose between three different reply options. They could opt for (a) rehabilitation without any direct access by footpaths along the creek (maximum protection); (b) no footpaths along the creek, but a limited number of specific access points (compromise between protection and access); or (c) full access to the rehabilitated site provided by newly created footpaths along the creek (maximum access). Given this choice almost one third, 29.8 per cent, of the respondents opted for (c), 50.2 per cent for (b) and only 20.0 per cent for (a). This indicates that despite a positive attitude towards nature protection in general, most people still want to have personal access to what they consider as 'nature' even though this is obviously not the best solution from an ecological point of view. The more access provided, the more the ecosystems will be disturbed and degraded. This somewhat janus-faced public opinion and behaviour is also visible when it comes to pets. Dogs are considered as a major problem in nature reserves and rehabilitated areas, because they disturb wildlife and even chase away native animals (especially birds, but also reptiles, amphibians and small mammals). For this reason dogs have to be leashed in nature reserves. A sample drawn from people who visited the rehabilitation areas on selected days $(\mathrm{N}=1,062)$, however, revealed that about one third of all the visitors was accompanied by dogs. 60 per cent of these dogs were not leashed. For this reason, it is no wonder that pet dogs and their owners are of great concern for the responsible authorities. It might be even necessary to exclude dogs completely from these areas to guarantee nature's protection.

\section{Conclusions}

Public participation is an integral part of ecological rehabilitation projects in Germany and other EU member states. Our research shows that local support to the Pulheim creek rehabilitation scheme is generally high. This is in line with the previous studies, which also indicate that a majority of people in Western Europe wants to see more natural and rehabilitated river ecosystems (Junker and Buchecker 2008; Buijs 2009; Schaich 2009). However, Connelly, Knuth and Kay (2002) found in their study on the Hudson River Estuary Action Plan in the USA that broad ecosystem restoration goals were more strongly supported than the corresponding specific implementation actions. This is somewhat contradictory to our findings which indicate that people's support for specific implementation projects in their local environment is even greater. This might have to do with cultural differences between the US and Western Europe, but most certainly more important is the scale of the projects. A small project scheme like the one described in this paper, is unlikely to cause major conflicts of use or access restrictions and is therefore easier to implement. Even though there is some criticism by the farmers with land adjoining the creek, this does not slow down the rehabilitation process to a considerable degree. Firstly, the rehabilitation projects concentrate on open land and stay out of builtup areas. Secondly, the 'Bachverband' (creek authority) buys the additional land necessary for plantings or meanders and from the private land owners (and it can do so because there is not much space involved). This small-scale participatory approach lowers conflict, but 
in the end leads to a somewhat fragmented pattern of ecological rehabilitation. As a result, many sections of the creek will remain virtually unchanged. This is a drawback from an ecological point of view, but also a necessary compromise when trying to rehabilitate a small creek in an area intensively used for farming activities and housing. Under these circumstances, a full ecological restoration will certainly remain a distant, unreachable goal. Rehabilitation of some crucial sections of the creek, however, is possible and can lead to some fundamental improvements, especially with regard to water quality, scenic values and recreational opportunities. This confirms the findings of a Swiss study by Junker and Buchecker 2008 which also indicate that even small rehabilitation efforts can make a positive difference to how people assess the rivers' aesthetic appearance and quality.

The careful and participatory approach adopted by the authorities is certainly an important success factor of the Pulheim creek rehabilitation scheme. Other decisive factors include favourable political constellations, especially the strong commitment of the chairman of the 'Bachverband', Horst Engel, which is also a member of the state parliament. Moreover, the former prime minister of the state of North Rhine-Westphalia, Jürgen Rüttgers, lives in Geyen (study area 3), which made it easier to acquire state money for the rehabilitation scheme.

While the findings are certainly favourable for rehabilitation projects overall, they also reveal critical points that environmental planners and managers should take into consideration: Firstly, though participation works overall, some segments of the population are harder to reach than others. This especially applies to young adults. They are significantly less informed and less interested than older people. The 'green classroom initiative', but also a co-operation between the Bachverband and the University of Cologne on research and tertiary teaching are steps into the right direction. However, innovative ways have to be paved to increase the interest of young people and to involve them into (local) rehabilitation projects.

Secondly, environmentalists' ideas prevail in the general public, but they are not unlimited and not unconditional. People want to have the opportunity to enjoy nature, they do not accept to be detached from it. Many people support rehabilitation projects, but they also want to be able to access the rehabilitated areas for recreational and leisure activities. While some of these activities might not seriously thwart ecological objectives, others do. Especially the disturbances of wildlife caused by pet dogs, many of them unleashed, are a major concern. This problem is hard to solve as people expect that access to public land is more or less unlimited and the concept of nature as consumer good prevails in postmodern consumer societies. Environmental managers have to find a delicate balance between ambitious ecological goals and people's demand for recreational space. This is not only true in Europe but in many other parts of the (developed) world.

Acknowledgements: We thank 42 geography students of Cologne University who participated in the questionnaire survey between September 2009 and June 2010. Without their commitment and assistance it would not have been possible to conduct so many interviews in such a short period of time. The students' suggestions and innovative ideas very much contributed to the success of our research. We are also indebted to Mr Horst Engel, the chairman of the Bachverband, who provided us organisational support wherever possible. Last but not least we have to thank our colleague Dr Reinhard Zeese, who has been involved in the Pulheim creek rehabilitation project for many years and who is the father of the cooperation between the Bachverband and the Geosciences Department of the University of Cologne, Germany.

\section{References}

Atchia M and Tropp S (eds.) 1995. Environmental Management: Issues and Solutions. John Wiley \& Sons, Chichester.

Boehmer HJ. 2011. Biologische Invasionen. Muster, Prozesse und Mechanismen der Bioglobalisierung. Geograph. Rundschau 63(3): 4-10.

Boon PJ, Davies, BR and Petts GE. 2000. Global Perspectives on River Conservation: Science, Policy, and Practice. John Wiley \& Sons, Chichester.

Box TW. 1978. The significance and responsibility of rehabilitating drastically disturbed land. In: Reclamation of Drastically Disturbed Lands (eds. Schaller FW and Sutton P), pp. 1-10. American Society of Agronomy, Madison, Wisconsin.

Bradshaw AD. 2002. Introduction and philosophy. In: Handbook of Ecological Restoration, Vol. 1 (eds. MR Perrow and AJ Davy), pp. 3-9. Cambridge University Press, Cambridge.

Buijs AJ. 2009. Public support for river restoration. A mixedmethod study into local residents' support for and framing of river management and ecological restoration in the Dutch floodplains. J. Environ. Management 90: 2680-2689.

Bundesamt für Naturschutz (BfN) 2006. Natursport und Kommunikation. BfN, Bonn.

Bundesamt für Naturschutz (BfN) 2010. FFHGebietsanmeldungen für Deutschland gemäß Art. 4 Abs. 1 der FFH-Richtlinie. BfN, Bonn. 
Bundesministerium für Umwelt, Naturschutz und Reaktorsicherheit (BMU) and Bundesamt für Naturschutz (BfN) 2010. Naturbewusstsein 2009. Bevölkerungsumfrage zu Natur und biologischer Vielfalt. BMU, Berlin, Bonn.

Cairns J. 2002. Rationale for restoration. In: Handbook of Ecological Restoration, Vol. 1 (eds. Perrow MRand and Davy AJ), pp. 10-23. Cambridge University Press, Cambridge.

Choi YD. 2004. Theories for ecological restoration in changing environment: toward 'futuristic' restoration. Ecol. Res. 19: 75-81.

Choi YD. 2007. Restoration ecology to the future: a call for new paradigm. Restor. Ecol. 15: 351-353.

Connelly NA, Knuth BA and Kay DL. 2002. Public support for ecosystem restoration in the Hudson River Valley, USA. Environ. Management 29: 467-476.

Davis MA. 2000. "Restoration” - a misnomer? Science 287: 1203.

Diggelen RV, Grootjans AP and Harris JA. 2001. Ecological restoration: state of the art or state of the science? Restor. Ecol. 9: 115-118.

European Union 2000. Directive 2000/60/EC of the European Parliament and of the Council 23 October 2000 Establishing a framework for community action in the field of water policy (Water Framework Directive). www.europa.eu/legislation_summaries/ agriculture/ environment/l28002b_en.htm.

Goodbody A. 2002. The Culture of German Environmentalism: Anxieties, Visions, Realities. Berghahn, New York.

Hobbs RJ. 2004. Restoration ecology: The challenge of social values and expectations. Ecol. Environ. 2: 43-44.

Holdgate M. 1996. From Care to Action - Making a Sustainable World. Earthscan Publications, London.

Junker B and Buchecker M. 2008. Aesthetic preferences versus ecological objectives in river restorations. Landsc. Urban Plan. 85: 141-154.

Leech S, Wiensczyk A and Turner J. 2009. Ecosystem Management: A Practioners' Guide. BC J. Ecosyst. Management 10(2): 1-12.
LNV (Dutch Ministry of Agriculture, Nature Management, and Fisheries) 1990. Nature Policy Plan of the Netherlands. LNV, The Hague, The Netherlands.

Loos GH and Schmitt T. 2011. Dynamik und Diversität invasiver Pflanzen in Deutschland. Geographische Rundschau 63 (3): 12-21.

Paavola J. 2004. Protected areas governance and justice: Theory and the European Union's Habitats Directive. Environ. Sci. 1: 59-77.

Schaich R. 2009. Local residents' perceptions of floodplain restoration measures in Luxembourg's Syr Valley. Landsc. Urban Plan. 93: 20-30.

Seeger H. 1999. The history of German waste water treatment. Euro. Water Management 2(5): 51-56

Shell D. (ed.) (2010). Jugend 2010: Eine pragmatische Generation behauptet sich. Fischer, Frankfurt am Main.

SER (Society for Ecological Restoration International Science and Policy Working Group) 2004. The SER Primer on Ecological Restoration, Version 2 (www.ser.org/ content/ ecological_restoration_primer.asp).

Van den Belt H. 2008. The local implementation of nature policy. In: Legitimacy in European Nature Conservation Policy - Case Studies in Multilevel Governance (eds. Keulartz J and Leistra G), pp. 227230. Springer, Heidelberg.

Wali MK. 1992. Ecology of the rehabilitation process. In: Ecosystem Rehabilitation (ed. Wali MK), pp. 3-26. SPB Academic Publishing, The Hague/Netherlands. Wali MK. 1999. Ecological succession and the rehabilitation of disturbed terrestrial ecosystems. Plant and Soil 213: 195-220.

Wurzel R. 2008. European Union environmental policy and Natura 2000. In: Legitimacy in European Nature Conservation Policy - Case Studies in Multilevel Governance (ed. Keulartz J. and Leistra G), pp. 259282. Springer, Heidelberg.

Manuscript received on 12 April 2011 and revised on 18 September 2011 